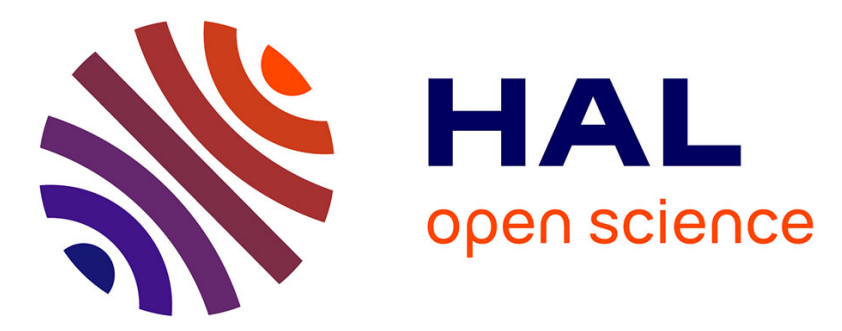

\title{
Influence de la variété et des zones de pression d'infection virale sur le taux de tubercules atteints par les virus et sur le rendement en culture de plant de pommes de terre en Pologne
}

W. Gabriel, L. Styszko, W. Woznica

\section{To cite this version:}

W. Gabriel, L. Styszko, W. Woznica. Influence de la variété et des zones de pression d'infection virale sur le taux de tubercules atteints par les virus et sur le rendement en culture de plant de pommes de terre en Pologne. Agronomie, 1987, 7 (6), pp.437-442. hal-00885012

\section{HAL Id: hal-00885012 \\ https://hal.science/hal-00885012}

Submitted on 1 Jan 1987

HAL is a multi-disciplinary open access archive for the deposit and dissemination of scientific research documents, whether they are published or not. The documents may come from teaching and research institutions in France or abroad, or from public or private research centers.
L'archive ouverte pluridisciplinaire HAL, est destinée au dépôt et à la diffusion de documents scientifiques de niveau recherche, publiés ou non, émanant des établissements d'enseignement et de recherche français ou étrangers, des laboratoires publics ou privés. 


\title{
Influence de la variété et des zones de pression d'infection virale sur le taux de tubercules atteints par les virus et sur le rendement en cul- ture de plant de pommes de terre en Pologne
}

\author{
W. GABRIEL, L. STYSZKO \& W. WOZNICA \\ Instytut Ziemniaka, Centre de Bonin, 76-009 Koszalin, Pologne
}

RÉSUMÉ

En Pologne, il y a 20 ans, 4 zones de pression d'infection des 2 principaux virus : le PLRV ef le PVY, ont été délimitées (fig. 1). Une étude est présentée ici maintenant, portant sur 3 séries de reproduction trisannuelle $\left(A_{1}-\right.$ $\left.A_{3}\right)$ de 6 variétés $\left(V_{1}-V_{6}\right)$ de résistance différente $\left(V_{3}-V_{7}\right)$ avec 3 traitements (épuration seule, prégermination et épuration, prégermination, épuration et défanage) (fig. 2).

L'infection est vérifiée partiellement par préculture et pour la totalité des localités par observation visuelle des essais aux champs où l'on a déterminé aussi le rendement (Re). Pour l'analyse statistique, on a utilisé la méthode de régression multiple.

Les équations de régression multiple du taux d'infection en données transformées sont présentées dans le texte : pour le PLRV, équations (1) à (3), pour le PVY, équations (4) à (6) et après détransformation, les résultats sont reproduits sur les figures 3 à 5 et le tableau 2 . Les données sur le rendement pour la moyenne des traitements sont calculées par l'équation (7) et présentées sur la figure 6.

En conclusion, il est conseillé de cultiver, dans différentes régions, des cultivars dont la résistance aux virus est adaptée à la pression d'infection (tabl. 3). Cette conclusion concerne en particulier les régions où le plant n'est pas renouvelé chaque année.

Mots clés additionnels : Virus $Y$, virus de l'enroulement.

Influence of potato cultivar and infection pressure on the percentage of virus-infected tubers and on seed-tuber yield in Poland.

\begin{abstract}
In Poland, 20 years ago, 4 infection pressure areas were defined for the 2 main viruses : PLRV and PVY (fig. 1). We report here on 3 experiments carried out over one to 3 years with 6 cultivars $\left(V_{1}-V_{6}\right)$ with different resistance rate $\left(\mathrm{Vc}_{3}-\mathrm{Vc}_{7}\right)$ and 3 treatments (roguing only, presprouting and roguing, presprouting, roguing and haulm removal) (fig. 2). Infection was checked by postharvest controls in some cases and for all tests by visual examination in field trials where yield was measured as well. For statistical analysis, the multiple linear regression method was used.

After data transformation, the multiple linear regression equations of infection rate are equations 1-3 for PLRV and 4-6 for PVY : after detransformation, results are reported in figures 3-5 and table 2. Equation 7 allowed us to calculate yield values for the average of treatments : these data are reported in figure 6 . In conclusion, it is advisable to grow in each region the cultivars that show an adapted virus-resistance level in relation to local infection pressure (table 3). This conclusion is particularly relevant to the regions where potato seed is not changed every year.
\end{abstract}

Additional key words : Potato virus Y (PVY), potato leafroll virus (PLRV).

\section{INTRODUCTION}

En Pologne, la pomme de terre est cultivée dans tout le pays sur plus de 2 millions d'hectares. Pour ensemencer ces surfaces, il faut de grandes quantités de plant pour la production desquelles, les régions propices à l'obtention d'un bon état sanitaire ne suffisent pas. De ce fait, l'agriculteur habitant les zones où la pression d'infection des virus est grande ne peut renouveler son plant tous les ans. 
En pratique, une grande partie du plant certifié doit être produite près des cultures de pommes de terre de consommation ou féculières pour éliminer les longs transports et approvisionner l'agriculteur à temps. En moyenne l'agriculteur ne peut renouveler son plant que tous les 2 à 5 ans en fonction des zones de pression d'infection.

Les recherches menées il y a 20 ans, ont permis de délimiter en Pologne 4 zones différant par la pression d'infection par le virus Y(PVY) et par le virus de l'enroulement (PLRV). Cette pression d'infection a été déterminée par le pourcentage de tubercules naturellement infectés dans des parcelles expérimentales cultivées pendant 4 ans dans environ 65 localités du pays. Une corrélation très significative ayant été mise en évidence entre la pression d'infection et les sommes des températures positives moyennes journalières (températures cumulées), les zones de pression ont été alors définies en fonction des températures cumulées depuis le $1^{\mathrm{er}}$ janvier jusqu'à une date fixée par calcul statistique. Ont été utilisées pour cela, les températures moyennes calculées sur une période de 15 ans (GABRIEL, 1965). Pendant ces 20 années, la pression de Myzus persicae Sulz. a augmenté fortement, alors que celle d'Aphis nasturtii Kalt. est restée à peu près constante (GABRIEL et al., 1981). Cela a fortement modifié le rapport PVY/PLRV. Si on ajoute aussi à cela qu'une réelle augmentation de la résistance des nouvelles variétés au virus $\mathrm{Y}$ a été obtenue, il n'est pas étonnant d'observer en moyenne une grande prédominance de l'infection par le virus de l'enroulement sur celle provoquée par le virus $Y$.

Ce n'est donc plus le virus $Y$ mais le virus de l'enroulement qui décide actuellement de l'état sanitaire des plantations du point de vue des maladies à virus. La figure 1 présente les zones de pression du virus de l'enroulement : dans la zone 1, la pression

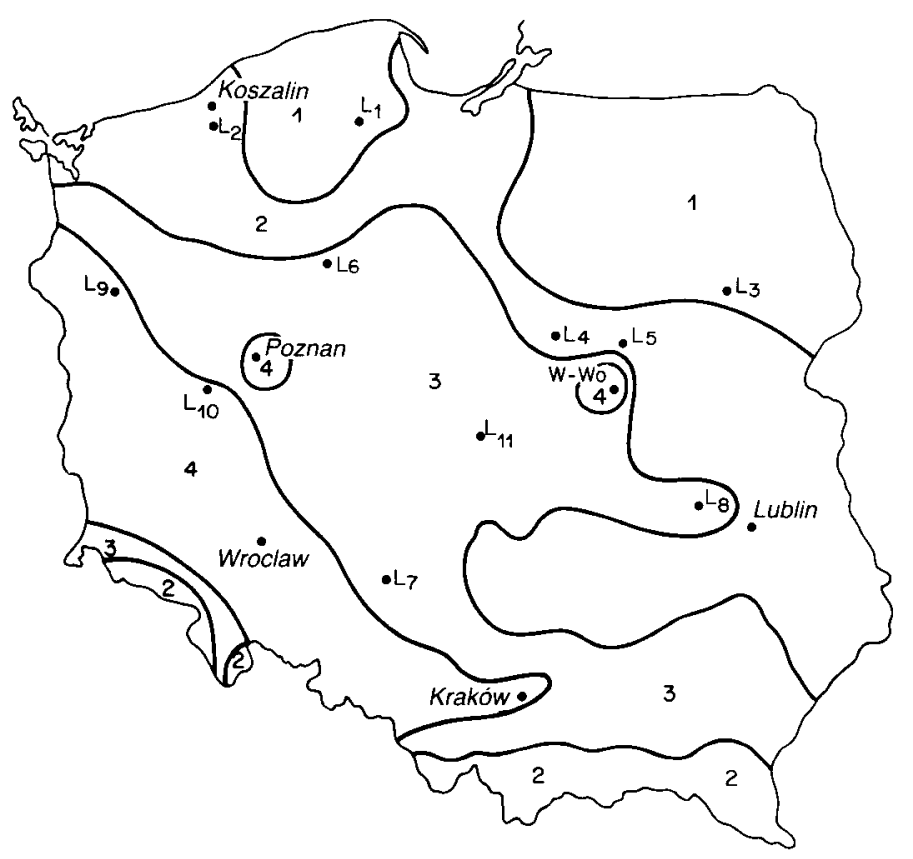

Figure 1

Zones de pression d'infection du virus de l'enroulement (1-4) en Pologne et localisation des essais $\left(l_{1}-l_{11}\right.$; voir le texte).

Regions of potato leaf roll infection pressure (1-4) and locations of trials $\left(l_{l}-l_{1 l}\right.$; see text). d'infection et des vecteurs est la plus faible, dans la zone 4 elle est la plus forte.

La situation décrite ci-dessus nous a incités à faire des recherches pour apprécier le niveau d'infection par les principaux virus ainsi que les pertes de rendement de variétés, de résistance différente, provoquées par la dégénérescence, dans les différentes zones.

\section{MATÉRIEL ET MÉTHODES}

Les essais effectués selon un même protocole ont eu lieu de 1970 à 1975 dans 11 localités réparties dans les 14 zones de pression (fig. 1). Ces localités sont les suivantes : Lubań $\left(l_{1}\right)$, Bonin $\left(l_{2}\right)$, Szepietowo $\left(l_{3}\right)$, Poświetne $\left(l_{4}\right)$, Jadwisin $\left(l_{5}\right)$, Minikowo $\left(l_{6}\right)$, Stare Olesno $\left(l_{7}\right)$, Końskowola $\left(l_{8}\right)$, Malyszyn $\left(l_{9}\right)$, Wielichowo $\left(l_{10}\right)$, Lućmierz $\left(l_{11}\right)$. Actuellement on a repris les résultats pour faire une nouvelle évaluation statistique. Trois séries de reproduction trisannuelles $(\mathrm{A} 1, \mathrm{~A} 2, \mathrm{~A} 3)$ ont été commencées en 1970, 1971 et 1972, en utilisant la première année du plant Elite. Le schéma d'une série est représenté sur la figure 2. Pour déterminer le rendement on a effectué, en plus, 2 autres séries, incomplètes, achevées en 1975 : une quatrième commencée en 1973 et une cinquième en 1974.

Pour les essais dans les 11 localités, en utilisant un plant Elite la première année, on a choisi 6 variétés

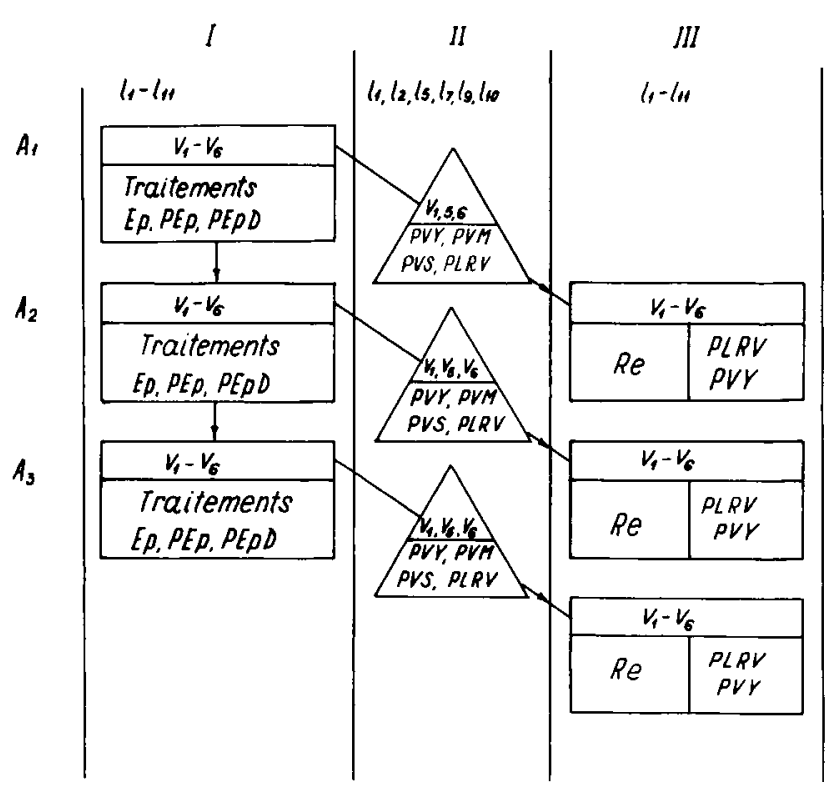

Figure 2

Schéma des essais. $l_{l}$ à $l_{1 l}$ : localisation des essais.

$A$ : années de reproduction. Ep : épuration. PEp : prégerminationépuration. PEpD: prégermination-épuration-défanage. $V_{l}-V_{6}$ : variétés de pomme de terre. Re : rendement. PVM : virus $M . P V S$ : virus $S$. PVY: virus $Y$. PLRV: virus de l'enroulement. I: expériences de base. $I I$ : indexage des tubercules + contrôle sérologique et test Solanum demissum « $Y$ ». III : contrôle au champ de la valeur de la semence.

Trial design. Site location l, yol.

A : Years of reproduction. Ep : roguing. PEp : presprouting + roguing. PEpD: presprouting + roguing + haulm removal. $V_{T^{-}}$ $V_{6}:$ potato cultivars. Re : yield. PVM: potato virus $M$. PVS: potato virus S. PVY: potato virus $Y$. PLRV : potato leafroll virus. $I$ : basic experiments. $I I:$ tuber indexing method combined with serological and Solanum demissum « $Y$ » tests. III : field assessment of seed value. 
$\left(V_{1}\right.$ à $\left.V_{6}\right)$ différant par leur précocité et leur indice de résistance au PLRV et PVY selon l'échelle en usage en Pologne et récemment décrite par CIEŚLEWICZ et al. (1983) : $9=$ immunité ; $8=$ grande résistance ; $1=$ moindre résistance. La résistance de ces variétés au PLRV et au PVY va de 3 à $7\left(\mathrm{Vr}_{3}-\mathrm{Vr}_{7}\right)$. Ces variétés sont les suivantes :

- variétés précoces : $V_{1}$ - « Giewont 》 (résistance 3 au PLRV et 5 au PVY). $V_{2}$ - « Pierwiosnek » (résistance 4 au PLRV et 6 au PVY) ;

- Variétés demi-précoces: $V_{3}$ - «Mittelfrühe Bóhm » (résistance 4 au PLRV et 3 au PVY). $V_{4}$ -« Epoka » (résistance 3 au PLRV et au PVY) ;

- variétés tardives $V_{5}$ - «Uran » (résistance $7 \mathrm{au}$ PLRV et 6 au PVY). $V_{6}$ - «Wyszoborski » (résistance 5 au PLRV et au PVY).

Chaque variété a subi 3 traitements dans les essais de base I :

- épuration seule

- prégermination, épuration

- prégermination, épuration et défanage (par arrachage des fanes)

Ep PEp

Dans chaque localité, l'année suivante, on a évalué dans les essais III le rendement ( $\mathrm{Re})$ et l'état sanitaire (observations visuelles) des tubercules récoltés dans les essais I.

Les données analysées concernant l'état sanitaire sont basées sur les 3 séries complètes (essais III : 19711973, 1972-1974, 1973-1975). Les différences de rendement entre $A_{1}$ et $A_{3}$ ont été déterminées en 1973, 1974 et 1975 en comparant les résultats des parcelles des séries, regroupées dans chaque localité en un seul essai.

Dans 6 localités $\left(l_{1}, l_{2}, 1_{5}, 1_{7}, 1_{9}, 1_{10}\right)$, des échantillons de 3 variétés $\left(V_{1}, V_{5}, V_{6}\right)$ ont été prélevés et testés, d'une part, en préculture (essais II) en serre avec écimage, d'autre part, par des tests sérologiques (précipitation) et, partiellement, biologiques sur Solanum demissum « $\mathrm{Y} »(\mathrm{Sd} \mathrm{Y})$.

Les essais I ont été réalisés avec 2 répétitions et 100 plantes par parcelle et les essais III avec 4 répétitions de 100 plantes. Les échantillons pour la préculture (essais II) étaient composés de 50 tubercules par parcelle.

Pour l'analyse statistique, le taux d'infection par le virus a été transformé par le « logit » de VANDERPLANK (1963) avec :

$$
\mathrm{Y}=\log \mathrm{x}(1-\mathrm{x})^{-1} \text {, }
$$

où $x$ est la fraction de tubercules infectés $(\mathrm{x} .100=\mathrm{p} \mathrm{p.} \mathrm{100)}$

Cette formule est bien adaptée pour normaliser les données relatives à des maladies polycycliques comme c'est le cas dans nos essais qui durant 3 ans concernent 3 cycles de contamination, à raison d'un cycle par an.

L'analyse statistique a été réalisée par la méthode de régression multiple du taux des tubercules infectés par le virus de l'enroulement et par le virus $Y$ ou du rendement par rapport aux zones de pression, sur la résistance des variétés et sur le nombre d'années de reproduction. L'introduction de 6 variables indépendantes pour le rendement et de 6 pour le virus, correspondant aux éléments cités ci-dessus et à leurs inter- actions a été nécessaire. Les équations de régression multiple n'utilisent que les variables qui augmentent la détermination d'une manière significative. La présentation des données sur les graphiques est faite après détransformation.

\section{RÉSULTATS}

\section{A. Influence de la zone de pression d'infection, de la résistance des variétés et du nombre d'années de reproduction sur le taux de plantes virosées dans les essais III}

Les taux moyens (moyenne géométrique) de plantes infectées par les virus en fonction des traitements et du nombre d'années de reproduction (tabl. 1) montrent bien, et ceci sera encore confirmé par la suite, que c'est le virus de l'enroulement qui décide en majeure partie de l'état sanitaire du plant. Pour l'analyse statistique, on a donc retenu les différentes zones de pression du virus de l'enroulement.

Les résultats $(\mathrm{N}=198)$ ont été analysés pour chaque traitement et les équations de régression multiple et les coefficients de corrélation multiple suivants ont été obtenus selon les traitements :

$$
\begin{aligned}
& \text { - pour le virus de l'enroulement (PLRV) : } \\
& \text { Ep : } Y_{L}=-0,777+1,023 Z_{L}+0,108 \mathrm{AZ}_{\mathrm{L}}-0,101 \mathrm{Vr}_{\mathrm{L}} \mathrm{Z}_{\mathrm{L}} \\
& \mathrm{R}=0,820 \\
& \text { PEp : } Y_{L}^{\prime}=-0,426 A+0,744 Z_{L}+0,250 A Z_{L}-0,108 \operatorname{Vr}_{L} Z_{L} \\
& \mathrm{R}=0,931 \\
& \text { PEpD : } Y_{L}^{\prime \prime}=-0,304 A+0,477 Z_{L}+0,178 A Z_{L} \\
& -0,0639 \mathrm{Vr}_{\mathrm{L}} Z_{\mathrm{L}} \\
& R=0,900
\end{aligned}
$$

- pour le virus Y (PVY) :

$$
\begin{gathered}
\text { Ep : } Y_{Y}=0,369 A+0,594 Z_{Y}-0,0599 A \operatorname{Ar}_{Y}-0,0687 \operatorname{Vr}_{Y} Z_{Y} \\
R=0,914 \\
\text { PEp : } Y_{Y}^{\prime}=0,803 A+0,727 Z_{Y}-0,103 V_{Y} Z_{Y} \\
R=0,904 \\
\text { PEpD : } Y_{Y}^{\prime \prime}=0,0537 A+0,583 Z_{Y}-0,0886 V_{Y} Z_{Y} \\
R=0,840
\end{gathered}
$$

où : $\mathrm{Y}=$ infection en données transformées.

A $=$ nombre d'années de reproduction: de 1 à 3 .

$\mathrm{Z}=$ zone de pression d'infection $\left(Z_{L}\right.$ à l'enroulement, $Z_{Y}$ au virus $Y$ ) : de 1 à 4 .

$\mathrm{Vr}_{\mathrm{L}}=$ résistance des variétés au PLRV de 3 à 7 .

$\mathrm{Vr}_{\mathrm{Y}}=$ résistance des variétés au PVY de 3 à 6 .

\section{TABLEAU}

Influence des traitements et du nombre d'années de reproduction (A) sur le taux moyen d'infection du plant des essais de base I (plantes des essais III).

Influence of treatments and number of years of reproduction $(A)$ on the mean percentage of seed infection in the basic experiments (I) (plants of tests III).

\begin{tabular}{lcccccc}
\hline \hline \multirow{2}{*}{ Traitement } & \multicolumn{6}{c}{ Moyenne géométrique $\%$} \\
& A-1 & \multicolumn{2}{c}{ A-2 } & \multicolumn{2}{c}{ A-3 } \\
& PVY & PLRV & PVY & PLRV & PVY & PLRV \\
\hline \multirow{2}{*}{ Ep } & 0,6 & 0,8 & 0,7 & 1,6 & 0,7 & 2,6 \\
PEp & 0,4 & 0,7 & 0,5 & 1,2 & 0,7 & 1,8 \\
PEpD & 0,3 & 0,4 & 0,3 & 0,6 & 0,4 & 0,8 \\
\hline \hline
\end{tabular}


La grande quantité de données oblige à simplifier la présentation des résultats. Dans le tableau 1, est présentée l'influence moyenne des années de reproduction et des traitements. Le traitement PEp et l'année A-2 donnent des résultats intermédiaires et pour une meilleure clarté des résultats, ceux-ci ne seront pas insérés dans les graphiques.

La représentation des taux d'infection par le PVY et le PLRV du plant après 3 ans de reproduction et lorsqu'il y a eu seulement épuration (fig. 3) indique

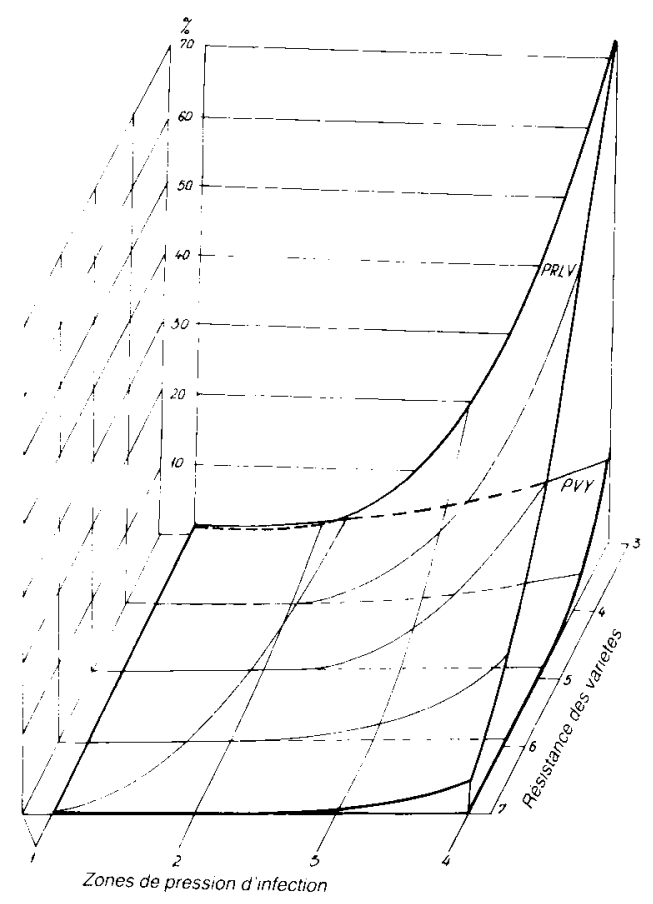

Figure 3

Infection par le virus $Y$ et le virus de l'enroulement des variétés de résistance différente, dans les 4 zones de pression d'infection après 3 années de reproduction.

$P V Y$ and PLRV infection of cultivars with different resistance rates in four infection pressure areas after three years of reproduction. qu'une grande différence existe entre l'infection par le PLRV et par le PVY, particulièrement dans les zones à pression d'infection élevée.

Le tracé des surfaces de régression qui représentent l'infection des tubercules par le virus de l'enroulement, par rapport à la pression d'infection dans différentes zones et par rapport à la résistance des variétés (fig. 4) montre la forte influence de ces facteurs et de leur interaction, ainsi que l'efficacité du défanage sur la limitation de l'augmentation du taux de tubercules atteints par le PLRV.

Sur la figure 5 sont rapportées les données analogues pour le PVY.

Le tableau 2 représente le taux calculé de plantes qui seraient attaquées par des viroses graves (PVY et PLRV) après 1 et 3 années de reproduction dans les 4 zones de pression pour des variétés théoriques qui auraient la même classe de résistance aux 2 virus. Une ligne brisée sépare les taux d'infection qui dépassent, pour A-1, les normes de préculture du plant d'origine et une autre pour A-3 celles de la classe B. Ces lignes donnent aussi une image de la résistance des variétés qui serait nécessaire à la production de plant dans chacune des zones de pression.

\section{B. Influence des zones de pression d'infection et de la résistance des variétés sur les baisses de rendement dans les essais III}

La différence entre les rendements fournis par le plant reproduit 1 fois (A-1) et 3 fois (A-3) a été analysée et les équations de régression multiple suivantes, avec leurs coefficients de corrélation, ont été obtenues :

$$
\begin{array}{ll}
\text { - pour la moyenne des traitements } & \\
\quad(\mathrm{Ep}+\mathrm{PEp}+\mathrm{PEpD}) 3^{-1}: & \\
\mathrm{Y}_{\mathrm{Re}}=0,577 \mathrm{Z}_{\mathrm{L}}^{2}-0,00872 \mathrm{Vr}_{\mathrm{L}}^{2} \mathrm{Z}_{\mathrm{L}}^{2} ; & \mathrm{R}=0,837 \\
- \text { pour le traitement Ep : } & \\
\mathrm{Y}_{\mathrm{Re}}^{\prime}=0,7424 \mathrm{Z}_{\mathrm{L}}^{2}-0,00120 \mathrm{Vr}_{\mathrm{L}}^{2} \mathrm{Z}_{\mathrm{L}}^{2} ; & \mathrm{R}=0,843
\end{array}
$$

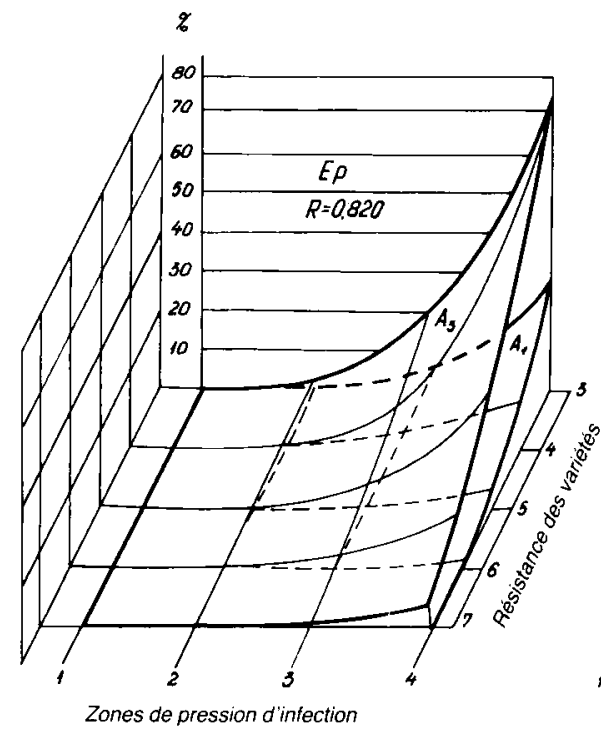

Figure 4

Infection par le virus de l'enroulement des variétés de résistance différente après une reproduction sous 2 traitements (Epuration : EP ; Prégermination + Epuration + Défanage : PEpD) pendant $I\left(A_{\jmath}\right)$ et $3\left(A_{3}\right)$ années, dans 4 zones de pression d'infection.

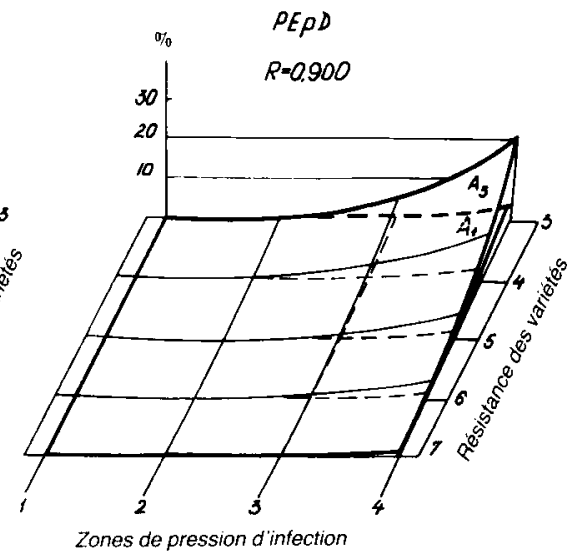

$P L R V$ infection of cultivars with different resistance rates, reproduced for $I\left(A_{l}\right)$ and $3\left(A_{3}\right)$ years with 2 treatments (Roguing: Ep ; Presprouting + Roguing + Haulm removal: PEpD) in 4 PLRV infection pressure areas. 

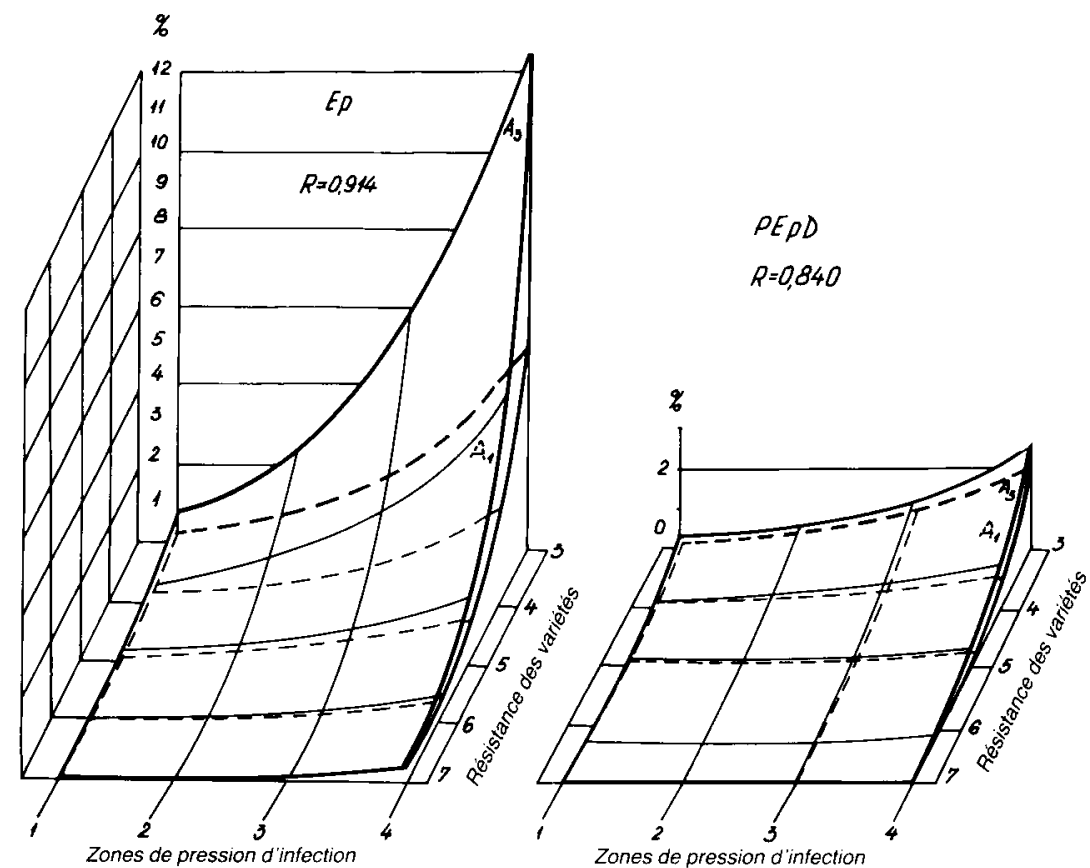

Figure 5

Infection par le virus $Y$ des variétés de résistance différente après une reproduction sous 2 traitements (Epuration : EP; Prégermination + Epuration + Défanage : PEpD) pendant $1\left(A_{l}\right)$ et $3\left(A_{3}\right)$ années, dans 4 zones de pression d'infection.

\section{TABLEAU 2}

Pourcentage calculé d'infection par les viroses graves (PLRV + $P V Y$ ) de plant de variétés " théoriques" (indices de résistance identiques pour le PLRV et $P V Y$ ) de résistance différente, après 1 an et 3 ans de reproduction, sans défanage, dans chacune des 4 zones de pression d'infection.

Calculated percentage of infection by severe virus diseases (PLRV and PVY) in "theoretical" cultivars (same group of resistance for $P L R V$ and $P V Y$ ) with various resistance rates, after 1 and 3 years of reproduction in each of the 4 zones of infection pressure.

\begin{tabular}{|c|c|c|c|c|c|}
\hline \multirow[t]{3}{*}{ Année } & \multirow{3}{*}{$\begin{array}{l}\text { Indice de } \\
\text { résistance }\end{array}$} & \multicolumn{4}{|c|}{$\begin{array}{c}\text { Pourcentage de virus (PLRV } \\
\text { PVY) }\end{array}$} \\
\hline & & \multicolumn{4}{|c|}{ Zones } \\
\hline & & 1 & 2 & 3 & 4 \\
\hline \multirow{5}{*}{ A-1 } & 3 & 0,4 & 1,6 & 6,8 & 29,6 \\
\hline & 4 & 0,2 & 1,0 & 3,6 & 13,9 \\
\hline & 5 & 0,2 & 0,6 & 1,8 & 6,0 \\
\hline & 6 & 0,1 & 0,4 & 1,0 & 2,4 \\
\hline & 7 & 0,1 & 0,2 & 0,5 & 0,9 \\
\hline \multirow{5}{*}{ A-3 } & 3 & 1,1 & 4,2 & 23,4 & 76,6 \\
\hline & 4 & 0,6 & 2,4 & 12,5 & 53,7 \\
\hline & 5 & 0,4 & 1,3 & 6,3 & 30,2 \\
\hline & 6 & 0,2 & 0,7 & 3,2 & 14,0 \\
\hline & 7 & 0,0 & 0,4 & 1,6 & 5,8 \\
\hline
\end{tabular}

- pour le traitement PEpD :

$\mathrm{Y}_{\mathrm{Re}}^{\prime \prime}=0,2404 \mathrm{Z}_{\mathrm{l}}^{2} \quad ; \quad \mathrm{R}=0,605$

où :

$\mathrm{Y}_{\mathrm{Re}}=$ rendement en $\mathrm{t} / \mathrm{ha}$.

$\mathrm{Z}_{\mathrm{L}}=$ zone de pression du PLRV de 1 à 4 .

Les différences de rendement calculées entre la première et la troisième année de reproduction d'après l'équation de régression (7) ont été divisées par 2 : la perte de rendement a pu ainsi être estimée pour une
$P V Y$ infection of cultivars with different resistance rates, reproduced for one $\left(A_{l}\right)$ and three $\left(A_{3}\right)$ years with 2 treatments (Roguing: $E p ;$ Presprouting + Roguing + Haulm removal: PEpD) in 4 $P L R V$ infection pressure areas.

année de reproduction (fig. 6). Sur le graphique, une ligne de pertes de rendement de $1 \mathrm{t} /$ ha correspondant à peu près à chaque ligne brisée $\left(A_{1}, A_{3}\right)$ du tableau 2 , a été tracée. Pour le traitement avec défanage $(\mathrm{PEpD})$, seule est significative la régression parabolique du rendement par rapport aux zones de pression. Ces pertes moyennes se chiffrent par année de reproduction pour la zone 1 à $0,1 \mathrm{t} / \mathrm{ha}$, pour la zone 2 à

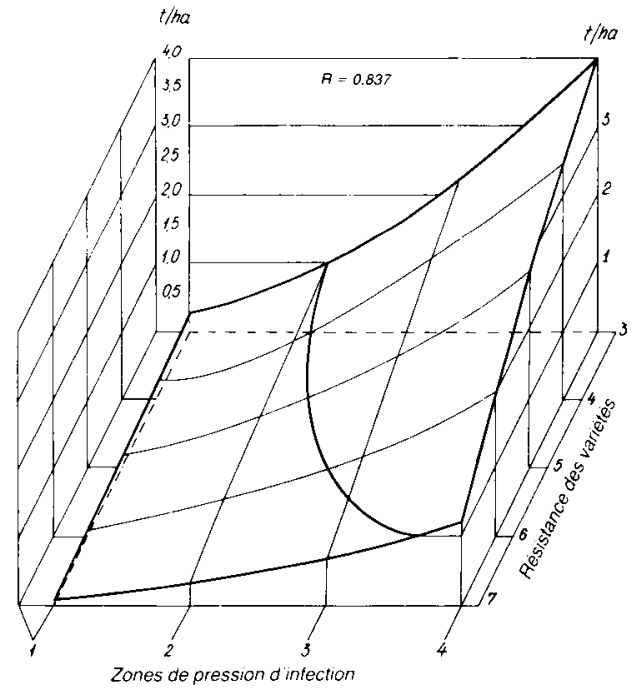

Figure 6

Baisse moyenne de rendement en une seule année de reproduction (moyenne de 3 traitements : EP, PEp, PEDD) en fonction de la résistance des variétés et de la zone de pression d'infection du virus de l'enroulement.

Mean yield decrease after a single year of reproduction (average of 3 treatments : Ep, $P E p, P E P D$ ) in relation to cultivar resistance and $P L R V$ infection pressure area. 
$0,5 \mathrm{t} / \mathrm{ha}$, pour la zone 3 à $1,1 \mathrm{t} /$ ha et pour la zone 4 à $1,9 \mathrm{t} / \mathrm{ha}$. Pour la zone 1 et partiellement pour la zone 2, les pertes sont plus grandes que sans défanage. En effet s'il n'est pas compensé par une amélioration de l'état sanitaire, le rendement du plant se trouve légèrement abaissé après défanage.

\section{DISCUSSION}

Les résultats des recherches confirment nettement l'influence très prononcée des zones de pression d'infection et démontrent celle de la résistance des variétés sur l'état sanitaire du plant et le rendement des pommes de terre.

\section{TABLEAU 3}

Limite de résistance de variétés destinées à la production de plant en fonction des zones de pression d'infection et du nombre d'années de reproduction.

Resistance limit of cultivars for seed production in relation to the zones of infection pressure and the number of years of reproduction.

\begin{tabular}{|c|c|c|c|c|}
\hline \multirow[t]{2}{*}{$\begin{array}{l}\text { Zone de } \\
\text { pression } \\
\text { du PLRV }\end{array}$} & \multicolumn{4}{|c|}{$\begin{array}{cc}\text { Limites de résistance de variétés }{ }^{*} \\
\text { Ep } & \text { PEpD } \\
\mathrm{Nb} \text { d'années } & \text { Nb d'années } \\
\text { de reproduction } & \text { de reproduction }\end{array}$} \\
\hline & 3 & 1 & 3 & 1 \\
\hline 1 & sans & sans & sans & sans \\
\hline 2 & 3 & sans & sans & sans \\
\hline 3 & 5 & 4 & 3 & 3 \\
\hline 4 & 7 & 6 & 5 & 4 \\
\hline
\end{tabular}

* : : Indice de résistance (cf. texte).

Ep : Epuration seule.

PEpD : Prégermination, épuration et défanage.
A partir des données résumées dans le tableau 3, issues de l'interprétation du tableau 2, on peut déduire que pour éviter le déclassement, il faut produire des variétés pour lesquelles la résistance dépasse 3 dans la zone 2 ; 5 dans la zone 3 et devrait dépasser 7 dans la zone 4. Ces données sont surtout valables pour le PLRV. La résistance de la majorité des variétés polonaises au PVY est suffisante. Pour les variétés limites, les pertes moyennes annuelles ne dépassent pas $1 \mathrm{t} / \mathrm{ha}$. L'agriculteur qui achètera ce plant pourra avoir des pertes plus grandes, mais il aura quand même la possibilité de réussir sa culture.

Le plant de base est produit dans les régions à pression d'infection faible. On y cultive aussi du plant certifié de variétés moins résistantes car ces variétés subissent, d'année en année, des pertes trop importantes lorsqu'elles sont cultivées en zones de grande pression. Cependant, dans ces mêmes zones, pour la production du plant, on pourrait probablement abaisser notablement la limite, actuellement nécessaire, de résistance des variétés si on introduisait certains traitements non appliqués jusqu'ici en Pologne pour le plant certifié, à savoir le défanage précoce et les insecticides. Cependant, ces variétés ne pourraient être utilisées ultérieurement par l'agriculteur que pendant 2 ans seulement pour' assurer la réussite de sa culture.

En conclusion, pour une production de pommes de terre à grande échelle, dans un pays comme la Pologne où le climat accentue fortement les différences d'état sanitaire du plant dans les différentes régions, ou dans tout autre pays analogue, l'introduction des variétés résistantes aux virus revêtira une importance extrême lorsqu'elles devront être cultivées dans les régions à pression d'infection de virus élevée. Cette conclusion concerne en particulier les régions où le plant n'est pas renouvelé chaque année.

Reç le 18 juin 1986. Accepté le 10 mars 1987.

\section{RÉFÉRENCES BIBLIOGRAPHIQUES}

Ciéslewicz I., Gabriel W., Ciskowska E., Woźnica W., 1983. Resistance of Polish potato cultivars to spraing. Biul. Inst. Ziemn., 29. 17-26 (en polonais ; rés. anglais).

Gabriel W., 1965. Influence of temperature on the spread of aphidborne potato virus diseases. Ann. appl. Biol., 56, 461-475.
Gabriel W., Kostiw M., Wislocka M., 1981. The occurrence of aphids on potato plants in different regions of the country. Roczn. Nauk Roln. ser. E., vol. 11, 137-146 (en polonais, res. anglais).

Vanderplank J. E., 1963. Plant diseases. Epidemic and control. Acad. Press, New York. 